\title{
Triterpenoid Saponins from Albizia mollis
}

\author{
Zhong-Quan Cheng, ${ }^{\star} \S$ Dan Yang, ${ }^{\S}$ Qing-Yun Ma, ${ }^{\dagger}$ Xin-Hui Yi, ${ }^{\S}$ Nen-Ling Zhang, Jun Zhou, ${ }^{*, * *}$ and You-Xing Zhao ${ }^{\dagger, *, *}$ \\ †Institute of Tropical Bioscience and Biotechnology, Chinese Academy of Tropical Agricultural Sciences, \\ Haikou 571101, P. R. China. "E-mail: zhaoyx1011@163.com \\ :State Key Laboratory of Phytochemistry and Plant Resources in West China, Kunming Institute of Botany, \\ the Chinese Academy of Sciences, Kunming 650204, P. R. China \\ ${ }^{\S}$ Guilin Normal College, Guilin 541001, P. R. China \\ Received February 19, 2011, Accepted February 22, 2011
}

Key Words : Albizia mollis, Saponins, Molliside A, Molliside B

Plant species of Albiziaceae are known for their traditional medicinal uses as medicaments of mind unrest, insomnia, physique damage, carbuncle gall in many parts of tropic zone. Albizia mollis, popularly named "maoyehehuan" in China, is one of representative plant species of this family. This plant is well known for its sedative and sleeping pill properties. $^{1}$ The previous chemical investigation of this family has led to the isolation of lignans, ${ }^{2-5}$ flavonoids, ${ }^{6-9}$ saponins, ${ }^{10}$ alkaloids, ${ }^{11}$ together with other secondary metabolites. ${ }^{12}$ In search for new and bioactive compounds from medicinal plant, the chemical study on A. mollis, collected from Kunming Botanical Garden, Yunnan Province, Peoples Republic of China, led to the isolation of four saponins including two new ones. This paper describes the isolation and structural elucidation of one new bisdesmosidic triterpene glycoside (molliside A, 1) and one new trisdesmosidic triterpene glycoside (molliside B, 2), together with two known compounds concinnoside A (3), albiziasaponin A (4).

Molliside A (1) was isolated as white powder. Its molecular formula was determined to be $\mathrm{C}_{42} \mathrm{H}_{66} \mathrm{O}_{13}$ with ten unsaturation degrees by HRESIMS ( $\left.m / z 777.4428[\mathrm{M}-1]^{-}\right)$and ${ }^{13} \mathrm{C}$ NMR spectra. The ${ }^{1} \mathrm{H}$ NMR spectrum of compound 1 clearly showed eight methyls at $\delta 0.86(3 \mathrm{H}, \mathrm{s}), 0.92(3 \mathrm{H}, \mathrm{s}), 0.95$ $(3 \mathrm{H}, \mathrm{s}), 1.00(3 \mathrm{H}, \mathrm{s}), 1.00(3 \mathrm{H}, \mathrm{s}), 1.06(3 \mathrm{H}, \mathrm{s}), 1.24(3 \mathrm{H}, \mathrm{s})$, $1.26(\mathrm{~d}, J=6.5 \mathrm{~Hz})$, two anomeric protons of $\beta$-type sugar at $\delta 4.30(1 \mathrm{H}, \mathrm{d}, J=7.0 \mathrm{~Hz}), 4.31(\mathrm{~d}, J=6.0 \mathrm{~Hz})$ and one olefinic proton at $\delta 5.42(1 \mathrm{H}, \mathrm{s})$. The ${ }^{13} \mathrm{C}$ NMR (DEPT) spectroscopic data (Table 1 ) revealed the presence of 8 methyls, 9 methylenes, 17 methines and 8 quaternary carbons. The IR absorption band at $1766 \mathrm{~cm}^{-1}$ and the peak at $\delta 181.6$ in the ${ }^{13} \mathrm{C}$ NMR spectrum showed the presence of $\gamma$-lactone ring. The gross structure of the aglycone of 1 was determined by analysis of NMR data and by referring to the NMR data of acacic acid lactone. ${ }^{13}$ The formation of lactone ring with the carboxyl group at C-17 was confirmed by the key HMBC correlation of H-21 ( $\delta$ 4.26) with carboxyl carbon $(\mathrm{C}-28, \delta$ 181.6). In the ROESY experiment, the key correlations of $\mathrm{H}-16 / \mathrm{H}-26, \mathrm{H}-16 / \mathrm{H}-18$ indicated $\beta$-orientations of $\mathrm{H}-16, \mathrm{H}-18$ and H-3/H-23, H-3/H-5, H-5/H-9, H-21/H-29 indicated $\alpha$-orientations of $\mathrm{H}-3, \mathrm{H}-5, \mathrm{H}-9$ and $\mathrm{H}-21$, from which the relative configuration of the aglycone in $\mathbf{1}$ was elucidated (see Figure 3).
Comparing the NMR data of 1 with those of compound $\mathbf{3}^{14}$ indicated that $\mathbf{1}$ was similar to $\mathbf{3}$ except for one rhamnose unit in 1 replacing the arabinose in the literature. The linkages of interglycosides and the saccharide part to the aglycone were respectively established by the key HMBC correlations of H-1" $(\delta 4.30)$ with C-6' $(\delta$ 68.0) and H-1' $(\delta$ $4.31)$ with C-3 $(\delta 89.0)$. Moreover, the presence of rhamnose and glucose were further evidenced by the acid hydrolysis and the fragment ion peaks at $m / z 631$ [M-1-Rha] ${ }^{-}, 469$ [M$1-$ Rha-Glc] $]^{-}$in the negative FABMS spectrum of 1 . Based on the above evidences, compound 1 was identified as acacic acid lactone $3-O-\beta$-L-rhampyranosyl- $(1 \rightarrow 6)-\beta$-D-glucopyranoside, named molliside $\mathrm{A}$.

Molliside B (2) was established a molecular formula of $\mathrm{C}_{47} \mathrm{H}_{74} \mathrm{O}_{17}$ determined from a pseudomolecular ion peak at $m / z 909.4863[\mathrm{M}-\mathrm{H}]^{-}$by the HRESIMS. All the ${ }^{1} \mathrm{H}$ and ${ }^{13} \mathrm{C}$ NMR signals of its aglycone moiety were consistent with the corresponding resonances of $\mathbf{1}$. The relative configuration of the aglycone in $\mathbf{2}$ was same as $\mathbf{1}$ with $\beta$-orientations of $\mathrm{H}-16$ and $\mathrm{H}-18$ and $\alpha$-orientations of $\mathrm{H}-3, \mathrm{H}-5, \mathrm{H}-9$ and $\mathrm{H}-21$ and were elucidated by ROESY experiment. Comparing the NMR data of $\mathbf{2}$ with those of $\mathbf{1}$ indicated that the distinct difference between compounds $\mathbf{1}$ and $\mathbf{2}$ was the additional chemical shift at $\delta 107.0,77.4,72.6,71.7$, and 69.8 which are combined to be one xylose (see Table 1). The linkages of interglycosides were determined by key HMBC correlations from H-1"' ( $\delta 4.46)$ of xlyopyranosyl to C-4" ( $\delta$ 78.1) of rhampyranosyl and from H-1" ( $\delta 4.30)$ of rhampyranosyl to C-6' ( $\delta$ 67.3) of glucose. The linkage of the saccharide part to the aglycone were established by the key HMBC correlation of $\mathrm{H}-1$ ' $(\delta 4.31)$ with $\mathrm{C}-3(\delta 89.4)$. Furthermore, the presence of xylose, rhamnose and glucose were further evidenced by the acid hydrolysis and the fragment ion peaks

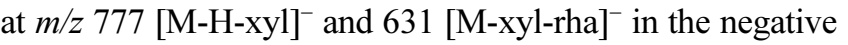
FABMS spectrum of 2 .

Based on the above evidences, the structure of $\mathbf{2}$ was determined to be as acacic acid lactone 3-O- $\beta$-D-xylopyranosyl-( $1 \rightarrow 4)-\beta$-L-rhampyranosyl-( $1 \rightarrow 6)-\beta$-D-glucopyranoside, named molliside $B$.

Two known compounds $\mathbf{3}$ and $\mathbf{4}$ had the similar spectral characteristic as triterpene glycosides $\mathbf{1}$ and $\mathbf{2}$ with the same aglycone. The spectral data of compound $\mathbf{3}$ were consistent 
Table 1. ${ }^{1} \mathrm{H}$ and ${ }^{13} \mathrm{C}$ NMR spectroscopic data for molliside A (1) and molliside B (2) (400 and $100 \mathrm{MHz}$, respectively, $\left.\mathrm{CD}_{3} \mathrm{OD}\right)$

\begin{tabular}{|c|c|c|c|c|}
\hline \multicolumn{3}{|r|}{1} & \multicolumn{2}{|r|}{2} \\
\hline & $\delta \mathrm{C}$ & $\delta \mathrm{H}$ & $\delta \mathrm{C}$ & $\delta \mathrm{H}$ \\
\hline 1 & 38.2 & $1 \mathrm{H}, 1.00, \mathrm{~m} ; 1 \mathrm{H}, 1.61, \mathrm{~m}$ & 39.7 & $1 \mathrm{H}, 1.11, \mathrm{~m} ; 1 \mathrm{H}, 1.63, \mathrm{~m}$ \\
\hline 2 & 25.6 & $2.18, \mathrm{~d}, 12 \mathrm{~Hz}$ & 27.2 & $1 \mathrm{H}, 1.54, \mathrm{~d}, 9.2 \mathrm{~Hz} ; 1 \mathrm{H}, 1.95, \mathrm{~d}, 6.7 \mathrm{~Hz}$ \\
\hline 3 & 89.0 & $2.32, \mathrm{~m}$ & 89.4 & $3.34, \mathrm{~d}, 4.4 \mathrm{~Hz}$ \\
\hline 4 & 36.3 & - & 37.9 & - \\
\hline 5 & 55.6 & $0.80, \mathrm{t}, 11 \mathrm{~Hz}$ & 57.1 & $0.82, \mathrm{~m}$ \\
\hline 6 & 17.7 & $1 \mathrm{H}, 1.51, \mathrm{~m} ; 1 \mathrm{H}, 1.61, \mathrm{~m}$ & 19.2 & $1.50, \mathrm{~m}$ \\
\hline 7 & 31.8 & $1.53, \mathrm{~m}$ & 33.4 & $1.52, \mathrm{~m}$ \\
\hline 8 & 39.8 & - & 41.4 & - \\
\hline 9 & 46.8 & $1.54, \mathrm{~m}$ & 48.4 & $1.57, \mathrm{~m}$ \\
\hline 10 & 38.6 & - & 40.3 & - \\
\hline 11 & 23.0 & $1 \mathrm{H}, 1.95, \mathrm{~m} ; 1 \mathrm{H}, 0.97, \mathrm{~m}$ & 24.6 & $1.96, \mathrm{~m}$ \\
\hline 12 & 124.5 & $5.42, \mathrm{~s}$ & 126.0 & $5.41, \mathrm{~s}$ \\
\hline 13 & 139.1 & - & 140.7 & - \\
\hline 14 & 42.8 & - & 44.3 & - \\
\hline 15 & 36.6 & $1 \mathrm{H}, 2.04, \mathrm{dd}, 5.0 \mathrm{~Hz}, 12.1 \mathrm{~Hz} ; 1 \mathrm{H}, 1.11, \mathrm{~m}$ & 38.2 & $1 \mathrm{H}, 1.14, \mathrm{~m} ; 1 \mathrm{H}, 2.03, \mathrm{~m}$ \\
\hline 16 & 66.4 & $1 \mathrm{H}, 3.90, \mathrm{dd}, 5.0 \mathrm{~Hz}, 12.1 \mathrm{~Hz}$ & 68.0 & $3.90, \mathrm{dd}, 5.2 \mathrm{~Hz}, 12.4 \mathrm{~Hz}$ \\
\hline 17 & 49.2 & - & 50.7 & - \\
\hline 18 & 41.2 & $2.51, \mathrm{~m}$ & 42.7 & $2.50, \mathrm{dd}, 6.4 \mathrm{~Hz}, 12.0 \mathrm{~Hz}$ \\
\hline 19 & 42.8 & $1 \mathrm{H}, 1.37, \mathrm{~m} ; 1 \mathrm{H}, 1.81, \mathrm{t}, 13.5 \mathrm{~Hz}$ & 44.3 & $1 \mathrm{H}, 1.38, \mathrm{~m} ; 1 \mathrm{H}, 1.82, \mathrm{~m}$ \\
\hline 20 & 33.3 & - & 34.9 & - \\
\hline 21 & 83.6 & $4.26, \mathrm{~m}$ & 85.2 & $3.34, \mathrm{~m}$ \\
\hline 22 & 27.2 & $2.18, \mathrm{~m} ; 2.33, \mathrm{~m}$ & 27.4 & $1 \mathrm{H}, 2.18, \mathrm{~d}, 12.0 \mathrm{~Hz} ; 1 \mathrm{H}, 2.32, \mathrm{~m}$ \\
\hline 23 & 26.9 & $1.06, \mathrm{~s}$ & 28.3 & $1.06, \mathrm{~s}$ \\
\hline 24 & 15.4 & $0.86, \mathrm{~s}$ & 17.0 & $0.86, \mathrm{~s}$ \\
\hline 25 & 14.6 & $0.95, \mathrm{~s}$ & 16.2 & $0.95, \mathrm{~s}$ \\
\hline 26 & 15.1 & $0.92, \mathrm{~s}$ & 16.7 & $0.92, \mathrm{~s}$ \\
\hline 27 & 27.7 & $1.24, \mathrm{~s}$ & 29.4 & $1.25, \mathrm{~s}$ \\
\hline 28 & 181.6 & - & 183.1 & - \\
\hline 29 & 27.4 & $1.00, \mathrm{~s}$ & 29.0 & $1.00, \mathrm{~s}$ \\
\hline 30 & 22.6 & $1.00, \mathrm{~s}$ & 24.2 & $0.99, \mathrm{~s}$ \\
\hline Glc & & & Glc & \\
\hline $1^{\prime}$ & 105.2 & $4.31, \mathrm{~d}, 6.0$ & 106.5 & $4.31, \mathrm{~d}, 8.0$ \\
\hline $2^{\prime}$ & 74.1 & $3.20, \mathrm{~m}$ & 77.1 & $3.48, \mathrm{~m}$ \\
\hline $3^{\prime}$ & 76.5 & $3.30, \mathrm{~m}$ & 71.1 & $3.49, \mathrm{~m}$ \\
\hline $4^{\prime}$ & 73.5 & $3.45, \mathrm{~m}$ & 71.7 & $3.61, \mathrm{~m}$ \\
\hline $5^{\prime}$ & 75.3 & $3.40, \mathrm{~m}$ & 75.0 & $3.62, \mathrm{~m}$ \\
\hline $6^{\prime}$ & 68.0 & $1 \mathrm{H}, 3.73, \mathrm{~m} ; 1 \mathrm{H}, 4.70, \mathrm{~d}, 10 \mathrm{~Hz}$ & 67.3 & $1 \mathrm{H}, 3.25, \mathrm{~d}, 10.8 \mathrm{~Hz} ; 1 \mathrm{H}, 3.95$, dd, $5.2 \mathrm{~Hz}, 10.8 \mathrm{~Hz}$ \\
\hline Rha & & & Rha & \\
\hline $1 "$ & 103.6 & $4.30, \mathrm{~d}, 7.0 \mathrm{~Hz}$ & 103.8 & $4.30, \mathrm{~d}, 7.0 \mathrm{~Hz}$ \\
\hline $2^{\prime \prime}$ & 70.8 & $3.48, \mathrm{~m}$ & 75.7 & $3.48, \mathrm{~m}$ \\
\hline 3" & 69.9 & $3.32, \mathrm{~m}$ & 75.0 & $3.58, \mathrm{~m}$ \\
\hline $4 "$ & 71.5 & $3.58, \mathrm{~m}$ & 78.1 & $3.32, \mathrm{~m}$ \\
\hline $5^{\prime \prime}$ & 70.4 & $3.60, \mathrm{~m}$ & 72.6 & $3.60, \mathrm{~m}$ \\
\hline $6^{\prime \prime}$ & 15.2 & $3 \mathrm{H}, 1.26, \mathrm{~d}, 6.5 \mathrm{~Hz}$ & 16.8 & $3 \mathrm{H}, 1.26, \mathrm{~d}, 6.5 \mathrm{~Hz}$ \\
\hline & & & $X y l$ & \\
\hline $1 " '$ & & & 107.0 & $4.46, \mathrm{~d}, 7.2 \mathrm{~Hz}$ \\
\hline $2^{\prime \prime \prime}$ & & & 77.4 & $3.94, \mathrm{~m}$ \\
\hline $3 " '$ & & & 72.6 & $4.38, \mathrm{~m}$ \\
\hline 4"' & & & 71.7 & $3.61, \mathrm{~m}$ \\
\hline $5^{\prime \prime \prime}$ & & & 69.8 & $1 \mathrm{H}, 3.73, \mathrm{dd}, 6.4 \mathrm{~Hz}, 12.0 \mathrm{~Hz} ; 1 \mathrm{H}, 4.05, \mathrm{~d}, 12.0 \mathrm{~Hz}$ \\
\hline
\end{tabular}

with those of concinnoside A reported in the literature. ${ }^{14}$ The linkage of arabinose to glucose at C-6-Glc were evidenced

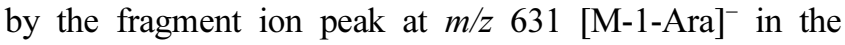
negative FABMS spectrum of $\mathbf{3}$ and the downfield shift of $\delta_{\mathrm{C}} 69.4$ (C-6-Glc). Base on above analysis, compound 3 was identified to be acacic acid lactone 3-O- $\beta$-D-arabinopyranosyl-( $1 \rightarrow 6)-\beta$-D-glucopyranoside (named concinnoside A). Compound $\mathbf{4}$ had the similar spectral data with those of albiziasaponin A reported in the literature. ${ }^{15}$ The linkages of xylose to arabinose at C-2-Ara and arabinose to glucose at 


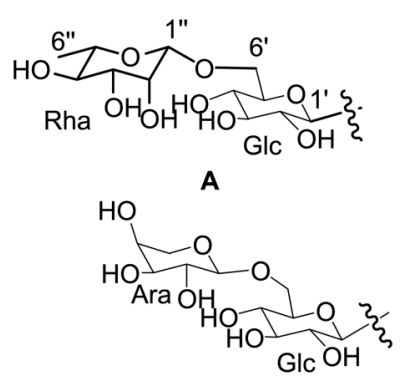

c

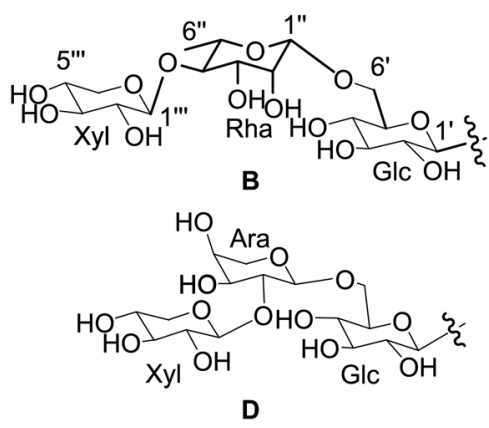

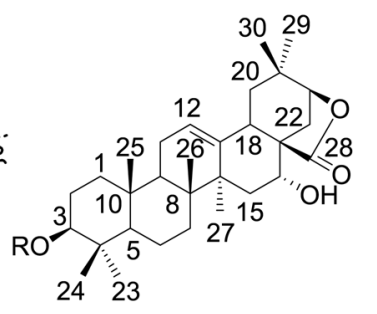

1234

$R=A B C D$

Figure 1. Structures of compounds 1-4.

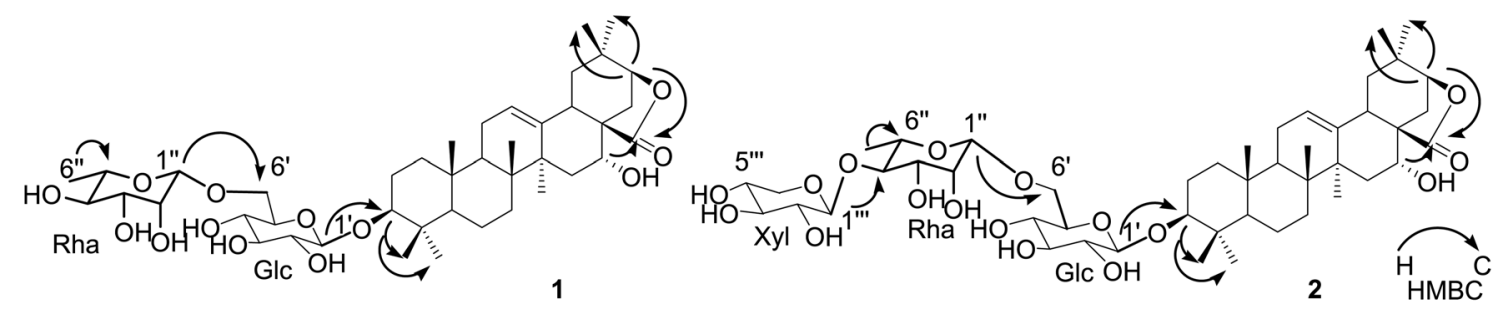

Figure 2. Key HMBC correlations of compounds $\mathbf{1}$ and $\mathbf{2}$.

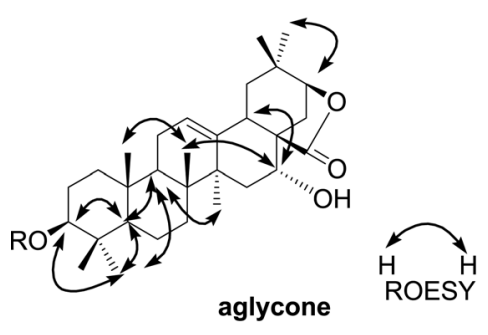

Figure 3. Key ROSEY correlations of aglycone.

C-6-Glc were further proposed by the fragment ion peak at $\mathrm{m} / z 763$ [M-1-Xyl] $^{-}$and 631 [M-1-Xyl-Ara $]^{-}$in the negative FABMS spectrum of $\mathbf{4}$ and the downfield shifts of $\delta_{\mathrm{C}} 81.4$ (C-2-Ara) and 69.5 (C-6-Glc). Thus, compound 4 was identified to be acacic acid lactone 3- $O$ - $\beta$-D-xylopyranosyl$(1 \rightarrow 2)-\beta$-L-arabinopyranosyl- $(1 \rightarrow 6)]-\beta$-D-glucopyranoside (named albiziasaponin $\mathrm{A}$ ).

\section{Experimental Section}

General Experimental Procedures. Melting points were measured on a XRC-1 micro-melting point apparatus and were uncorrected. MS spectra were obtained on a VG Auto Spec-3000 mass spectrometer. 1D and 2D NMR spectra were recorded on Bruker AM-400 MHz spectrometers, with chemical shifts $(\delta)$ in ppm relative to TMS as internal standard and coupling constants in hertz $(\mathrm{Hz})$. IR spectra were measured with a Bio-Rad FTS-135 spectrometer with $\mathrm{KBr}$ pellets. UV spectra were measured on a Hitachi UV3210 spectrophotometer. Silica gel (200-300 mesh) for column chromatography was product of the Qingdao Marine Chemical Ltd., Qingdao, P. R. China. Sephadex LH20 for chromatography was purchased from Amersham Biosciences. Reversed-phase chromatography was with RP-
18 (LiChroprep, 40-63 $\mu \mathrm{m}$, Merck, Darmstadt, Germany).

Plant Materials. The bark of $A$. mollis was collected in Kunming, Yunnan Province, Peoples Republic of China, in September 2008, and authenticated by Professor Xun Gong. A voucher specimen (ZM080920) has been deposited in the Herbarium of Kunming Institute of Botany, Chinese Academy of Sciences.

Extraction and Isolation. The air-dried and powdered bark of A. mollis $(18.0 \mathrm{~kg})$ were extracted three times each with $20 \mathrm{~L}$ of $95 \% \mathrm{EtOH}$ under reflux for $3 \mathrm{~h}$. The extracts were evaporated and the residue was resuspended in $15 \mathrm{~L}$ of $\mathrm{H}_{2} \mathrm{O}$ and partitioned successively with EtOAc $(5 \mathrm{~L} \times 3)$ and $n$-BuOH $(5 \mathrm{~L} \times 3)$ to yield EtOAc extract $(300 \mathrm{~g}), n-\mathrm{BuOH}$ extract (640 g), respectively. Part of $n$-BuOH extract (500 g) was applied to a silica gel column chromatography (200-300 mesh) eluted with $\mathrm{CHCl}_{3} / \mathrm{CH}_{3} \mathrm{OH} / \mathrm{H}_{2} \mathrm{O}(10: 3: 0.5, \mathrm{v} / \mathrm{v})$ to give five fractions. Fraction 1 (45 g) was purified by RP-18 with $10 \% \rightarrow 100 \%$ aqueous $\mathrm{CH}_{3} \mathrm{OH}$ and give five fractions. Sub-fraction 2 (96 mg) was purified on Sephadex LH-20 with $\mathrm{CH}_{3} \mathrm{OH} / \mathrm{CH}_{3} \mathrm{Cl} 1: 1$ and then subjected to semipreparative HPLC $\left(\mathrm{CH}_{3} \mathrm{OH} / \mathrm{H}_{2} \mathrm{O}, 62 / 38,2 \mathrm{~mL} / \mathrm{min}, 20{ }^{\circ} \mathrm{C}\right.$, detector wavelength $210 \mathrm{~nm})$, which gave compounds 2 (11 $\mathrm{mg}$ ) and compounds 4 (34 mg); Sub-fraction 3 (28 mg) was subjected to semipreparative HPLC $\left(\mathrm{CH}_{3} \mathrm{CN} / \mathrm{H}_{2} \mathrm{O}, 32 / 68,2\right.$ $\mathrm{mL} / \mathrm{min}, 20{ }^{\circ} \mathrm{C}$, detector wavelength $210 \mathrm{~nm}$ ) to give compounds 1 (6 mg) and $\mathbf{3}(10 \mathrm{mg})$.

Molliside A (1): White powder, mp 209-211 ${ }^{\circ} \mathrm{C} ;[\alpha]_{\mathrm{D}}^{21}$ $+6.3116^{\circ}\left(\mathrm{c} 0.500, \mathrm{CH}_{3} \mathrm{OH}\right)$; UV $\left(\mathrm{CH}_{3} \mathrm{OH}\right) \lambda_{\max }(\log \varepsilon): 191$ (3.519), 204 (3.712), 252 (3.260), 373 (2.508); IR (KBr) v 3425, 1766, 1602, 1063, $1045 \mathrm{~cm}^{-1}$; ${ }^{1} \mathrm{H}$ NMR (400 MHz, $\left.\mathrm{CD}_{3} \mathrm{OD}\right)$ and ${ }^{13} \mathrm{C}$ NMR (100 $\left.\mathrm{MHz}, \mathrm{CD}_{3} \mathrm{OD}\right)$ see Table 1; HRESIMS (negative ion) $\mathrm{m} / z$ 777.4428 [M-1] ${ }^{-}$(Calcd. for $\mathrm{C}_{42} \mathrm{H}_{65} \mathrm{O}_{13}, 777.4789$ ); FABMS (negative ion) $m / z$ (\%) 777 $[\mathrm{M}-1]^{-}$(100), 631 (3), 469 (3), 437 (5). 
Molliside B (2): White powder, mp 205-207 ${ }^{\circ} \mathrm{C},[\alpha]_{\mathrm{D}}^{21}$ $-24.8750^{\circ}$ (c $\left.0.320, \mathrm{CH}_{3} \mathrm{OH}\right) ; \mathrm{UV}\left(\mathrm{CH}_{3} \mathrm{OH}\right) \lambda_{\max }(\log \varepsilon)$ : 203 (3.892), 252 (3.005), 383 (2.194); IR (KBr) v 3423, $1762,1639,1165,1046 \mathrm{~cm}^{-1},{ }^{1} \mathrm{H}$ NMR $\left(400 \mathrm{MHz}, \mathrm{CD}_{3} \mathrm{OD}\right)$ and ${ }^{13} \mathrm{C}$ NMR (100 MHz, $\left.\mathrm{CD}_{3} \mathrm{OD}\right)$ see Table 1; HRESIMS (negative ion) $m / z 909.4863[\mathrm{M}-1]^{-}$(Calcd. for $\mathrm{C}_{47} \mathrm{H}_{73} \mathrm{O}_{17}$, 909.4847); FABMS (negative ion) $\mathrm{m} / z$ (\%) 909 [M-1 $^{-}$ (100), 777 (12), 631 (5), 437 (4).

Concinnoside A (3): white powder, ${ }^{1} \mathrm{H}$ NMR $(400 \mathrm{MHz}$, $\left.\mathrm{CD}_{3} \mathrm{OD}\right) \delta 0.85(3 \mathrm{H}, \mathrm{s}, \mathrm{H}-24), 0.86(3 \mathrm{H}, \mathrm{s}, \mathrm{H}-26), 0.92(3 \mathrm{H}$, $\mathrm{s}, \mathrm{H}-25), 0.99(3 \mathrm{H}, \mathrm{s}, \mathrm{H}-30), 1.00(3 \mathrm{H}, \mathrm{s}, \mathrm{H}-29), 1.06(3 \mathrm{H}, \mathrm{s}$, $\mathrm{H}-23), 1.24(3 \mathrm{H}, \mathrm{s}, \mathrm{H}-27), 4.26(1 \mathrm{H}, \mathrm{d}, J=7.8 \mathrm{~Hz}, \mathrm{H}-1-\mathrm{Ara})$, $4.31(1 \mathrm{H}, \mathrm{d}, J=6.9 \mathrm{~Hz}, \mathrm{H}-1-\mathrm{Glc}), 5.41(1 \mathrm{H}, \mathrm{s}, \mathrm{H}-12) ;{ }^{13} \mathrm{C}$ NMR $\left(100 \mathrm{MHz}, \mathrm{CD}_{3} \mathrm{OD}\right) \delta 37.9(\mathrm{t}, \mathrm{C}-1), 26.9(\mathrm{t}, \mathrm{C}-2), 85.2$ (d, C-3), 40.2 (s, C-4), 57.1 (d, C-5), 19.0 (t, C-6), 32.6 (t, C7), 40.2 (s, C-8), 48.4 (d, C-9), 37.8 (s, C-10), 24.5 (t, C-11), 126.1 (d, C-12), 140.1 (s, C-13), 44.3 (s, C-14), 37.9 (t, C15), 67.9 (d, C-16), 50.7 (s, C-17), 40.2 (d, C-18), $42.7(\mathrm{t}, \mathrm{C}-$ 19), 37.8 (s, C-20), 85.2 (d, C-21), 27.5 (t, C-22), 29.0 (q, C23), 16.2 (q, C-24), 15.2 (q, C-25), 16.8 (q, C-26), 27.0 (q, C-27), 183.1 (s, C-28), 29.2 (q, C-29), 24.1 (q, C-30), 106.8 (d, Glc. C-1), 75.5 (d, Glc. C-2), 78.0 (d, Glc. C-3), 71.6 (d, Glc. C-4), 76.7 (d, Glc. C-5), 69.4 (t, Glc. C-6), 105.0 (d, Ara. C-1), 72.4 (d, Ara. C-2), 74.1 (d, Ara, C-3), 67.9 (d, Ara. C-4), 66.4 (t, Ara. C-5); FAB-MS (negative ion) $\mathrm{m} / \mathrm{z}$ $763[\mathrm{M}-1]^{-}$(72), 631 (5), 347 (6).

Albiziasaponin A (4): white powder, ${ }^{1} \mathrm{H}$ NMR $(400 \mathrm{MHz}$, $\left.\mathrm{CD}_{3} \mathrm{OD}\right) \delta 0.86(3 \mathrm{H}, \mathrm{s}, \mathrm{H}-24), 0.91(3 \mathrm{H}, \mathrm{s}, \mathrm{H}-26), 0.92(3 \mathrm{H}$, s, H-25), 0.99 (3H, s, H-30), 1.00 (3H, s, H-29), 1.06 (3H, s, $\mathrm{H}-23), 1.26$ (3H, s, H-27), 4.32 (1H, d, $J=7.8 \mathrm{~Hz}, \mathrm{H}-1-\mathrm{Glc})$, $4.54(1 \mathrm{H}, \mathrm{d}, J=6.7 \mathrm{~Hz}, \mathrm{H}-1-\mathrm{Xyl}), 4.26(1 \mathrm{H}, \mathrm{d}, J=5.4 \mathrm{~Hz}$, $\mathrm{H}-1$-Ara), 5.40 (1H, s, H-12); ${ }^{13} \mathrm{C}$ NMR (100 MHz, $\left.\mathrm{CD}_{3} \mathrm{OD}\right)$ $\delta 38.0(\mathrm{t}, \mathrm{C}-1), 26.0$ (t, C-2), 89.8 (d, C-3), 38.2 (s, C-4), 57.1 (d, C-5), 19.1 (t, C-6), 32.8 (t, C-7), 41.3 (s, C-8), 48.3 (d, C9), 37.9 (s, C-10), $24.3(\mathrm{t}, \mathrm{C}-11), 125.9$ (d, C-12), $140.6(\mathrm{~s}$, C-13), 44.3 (s, C-14), 40.2 (t, C-15), 66.9 (d, C-16), 50.7 (s, C-17), 42.5 (d, C-18), 44.3 (t, C-19), 34.8 (s, C-20), 85.1 (d, C-21), 27.3 (t, C-22), 28.4 (q, C-23), 17.0 (q, C-24), 16.2 (q, C-25), 16.7 (q, C-26), 29.4 (q, C-27), 183.1 (s, C-28), 29.0 (q, C-29), 24.2 (q, C-30), 106.6 (d, Glc. C-1), 75.6 (d, Glc. C-2), 78.0 (d, Glc. C-3), 71.8 (d, Glc. C-4), 75.8 (d, Glc. C5), 69.5 (t, Glc. C-6), 103.3 (d, Ara. C-1), 81.4 (d, Ara. C-2), 72.8 (d, Ara, C-3), 67.8 (d, Ara. C-4), 66.5 (t, Ara. C-5), 106.6 (d, Xyl. C-1), 75.6 (d, Xyl. C-2), 77.5 (d, Xyl. C-3), 71.1 (d, Xyl. C-4), 67.5 (t, Xyl. C-5); FABMS (negative ion) $\mathrm{m} / \mathrm{z} 895$ [M-1] $]^{-}$(100), 763 (120), 631 (40).
Acid Hydrolysis of Compounds 1 and 2. Compounds 1 $(3 \mathrm{mg})$ and $2(3 \mathrm{mg})$ in $5 \% \mathrm{EtOH}(2 \mathrm{~mL})$ containing $5 \%$ $\mathrm{H}_{2} \mathrm{SO}_{4}$ was each heated in a boiling water-bath for $5 \mathrm{~h}$, then cooled for few minutes. The mixture was washed with EtOAc $(2 \mathrm{~mL} \times 2)$. The $\mathrm{H}_{2} \mathrm{O}$ layer was concentrated in vacuo to give a residue. The residue and authentic sugar were dotted to the plate developed with EtOAc/pyridine/ ethanol/ $\mathrm{H}_{2} \mathrm{O}(8 / 1 / 1 / 2, \mathrm{v} / \mathrm{v})$, and phenylene diamine-anilinephosphoric acid used as spray reagent, followed by heating at 80. From compounds $\mathbf{1}$ and $\mathbf{2}$ gulcose was detected, $R_{f}$. glucose 0.38 , xylose 0.55 , rhamnose 0.76 , respectively.

Acknowledgments. This research was supported by National Nonprofit Institute Research Grant of CATASITBB (No. 110301) and the fund (P2008-ZZ22) of State Key Laboratory of Phytochemistry and Plant Resources in West China. We thank the analytical group of the State Key Laboratory of Phytochemistry and Plant Resources in West China, Kunming Institute of Botany, Chinese Academy of Sciences for all spectra tests.

\section{References}

1. Committee of Flora of China, Flora of China; Science Press: Beijing, 1988; p 55.

2. Kinjo, J.; Fukui, K.; Higuchi, H.; Nohara, T. Chem. Pharm. Bull. 1991, 39, 1623

3. Kinjo, J.; Higuchi H, Fukui, K.; Nohara, T. Chem. Pharm. Bull. 1991, 39, 2952.

4. Higuchi, H.; Fukui, K.; Kinjo, J.; Nohara, T. Chem Pharm Bull. 1992, 40, 534

5. Ito, A.; Kasai, R.; Yamasaki, K.; Duc, N.; Nham, N. T. Phytochemistry 1994, 37, 1455.

6. Candy, H. A.; Brookes, K. B.; Bull, J. R.; McGarry, E. J.; McGarry, J. M. Phytochemistry 1978, 17, 1681.

7. El-Mousallamy, A. M. D. Phytochemistry 1998, 48, 759.

8. Pal, B. C.; Achari, B.; Yoshikawa, K.; Arihara, S. Phytochemistry 1995, 38, 1287.

9. Debella, A.; Haslinger, E.; Schmid, M. G.; Bucar, F.; Michl, G.; Abebe, D. Phytochemistry 2000, 53, 885.

10. Carpani, G.; Orsini, F.; Sisti, M.; Verotta, L. Phytochemistry 1989, $28,863$.

11. Rukunga, G. M.; Waterman, P. G. Phytochemistry 1996, 42, 1211.

12. Ueda, M.; Yamamura, S. Tetrahedron Lett. 1999, 40, 7823.

13. Zheng, L.; Wu, G.; Wang, B.; Wu, L. J. Journal of Peking University (Health Sciences) 2004, 36, 421.

14. Abdul, G. M.; Obata, T.; Kiuchi, F. Chem. Pharm. Bull. 1999, 45, 620.

15. Bikas, C. P.; Basudeb. A.; Kazuko, Y.; Shigenobu, A. Phytochemistry 1995, 38, 1287. 